\title{
eJRIEPS
}

Ejournal de la recherche sur l'intervention en éducation physique et sport

23 | 2011

Varia

\section{La démarche technologique en STAPS : analyse conceptuelle et mise en perspective pour les sports collectifs}

\section{Serge Eloi et J-F Robin}

\section{(2) OpenEdition}

1 Journals

Édition électronique

URL : http://journals.openedition.org/ejrieps/4540

DOI : $10.4000 /$ ejrieps. 4540

ISSN : 2105-0821

Éditeur

ELLIADD

Référence électronique

Serge Eloi et J-F Robin, « La démarche technologique en STAPS : analyse conceptuelle et mise en perspective pour les sports collectifs », eJRIEPS [En ligne], 23 | 2011, mis en ligne le 01 avril 2011, consulté le 21 février 2020. URL : http://journals.openedition.org/ejrieps/4540 ; DOI : 10.4000/ejrieps. 4540

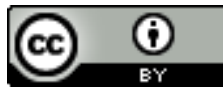

La revue eJRIEPS est mise à disposition selon les termes de la Creative Commons Attribution 4.0 International License. 
La démarche technologique en STAPS : analyse conceptuelle et mise en

perspective pour les sports collectifs.

Serge Eloi ${ }^{*}$ et Gilles Uhlrich**

* Université Paris-Est Créteil Val-de-Marne, Laboratoire REV/CIRCEF, 61 avenue du Général de Gaulle 94010, Créteil Cedex, France

** Université Paris-Sud 11, UFR STAPS, UR CIAMS/ECAMS, Bâtiment 335, 91405 Orsay Cedex, France

Résumé

Méthode originale du fait qu'elle est entièrement mise au service de l'intervenant, la démarche technologique doit permettre de structurer les projets d'intervention quel que soit leur secteur d'application (scolaire, fédéral ou de loisirs). La technologie est donc envisagée ici comme un " usage rationnel des techniques dans le cadre de la conception, la mise en œuvre et l'évaluation d'un projet d'intervention sur le réel. » (Bouthier, 2005). C'est nous semble-t-il à ce niveau que la jonction peut s'opérer entre technologie et intervention. Mais cette liaison nécessite l'abandon d'une conception figée de la technique. Après avoir fait un bref rappel des notions de registres (Ulrich et Éloi, dans ce numéro), nous nous attacherons à définir le concept d'artefact. II est classiquement défini comme un objet plus ou moins complexe ayant reçu une transformation d'origine humaine. Pour le définir, nous tenterons de dépasser les notions de phénomène, d'artifice, de gadget voire de parasite. Nous illustrerons notre propos en prenant en exemple les travaux que René Deleplace a développés pour les sports collectifs. II apparaît en effet que cet auteur a adopté une véritable démarche technologique alors même que le concept n'était pas encore évoqué.

\section{Introduction}

L'augmentation du nombre des recherches en technologie des Activités Physiques Sportives et Artistiques (APSA) (voir Mouchet, Amans-Passaga \& Gréhaigne pour une revue de littérature, 2010), notamment dans le cadre de l'étude des sports collectifs, nous engage à développer un cadre d'analyse qui permette d'ordonner les différentes approches. D'abord, il s'agit de caractériser la nature des différents travaux afin de les mettre en perspective les uns par rapport aux autres. Ensuite, il convient de définir ce que peut être un enseignement en technologie des APSA. Car il apparaît que des enjeux 


\section{eJRIEPS 23 avril 2011}

importants sont liés à la diffusion de ce type de savoirs. En effet, la caractéristique majeure de cette approche est de produire des connaissances répondant aux besoins des secteurs sociaux de pratique corporelle tout en prenant en compte les problèmes de terrain de ces secteurs sociaux comme objet central d'étude (Bouthier, 2008 a).

Cet article propose une modélisation de la démarche technologique en Sciences et Techniques des Activités Physiques et Sportives (STAPS). Dans une première partie, les notions de registres de technicité qui ont été décrites précédemment (cf. dans ce même numéro Ulrich \& Eloi) nous conduiront à développer le concept d'artefact. Notre objectif est de montrer comment il est possible d'envisager l'articulation entre registres de technicité et artefacts. Une telle visée conduit à rendre envisageable la spécification des recherches en technologie des Activités Physiques Sportives et Artistiques (APSA). Ainsi, il devient possible de caractériser les différents travaux en stipulant le registre principalement concerné et le type d'artefact(s) mobilisé(s). Dans une seconde partie, nous illustrerons notre propos en prenant en exemple les travaux que René Deleplace a développés. II apparaît en effet que cet auteur a adopté une véritable démarche technologique alors même que le concept n'était pas encore évoqué. En prenant appui sur ses travaux, il s'agira de déterminer ce qui ressort d'une modélisation des sports collectifs. Pour conclure, nous développerons des pistes permettant de préciser le processus de la démarche technologique en vue de l'élaboration de contenus de formation et de dispositifs d'enseignement en STAPS.

\section{La technologie des APSA}

Méthode originale du fait qu'elle est entièrement mise au service de l'intervenant, la démarche technologique doit permettre de structurer les projets d'intervention quel que soit leur secteur d'application (scolaire, fédéral ou de loisirs).

\section{1. L'intervention dans le champ des APSA}

Comprise sous cet angle, la technologie est donc envisagée comme un « usage rationnel des techniques dans le cadre de la conception, la mise en œuvre et l'évaluation d'un projet d'intervention sur le réel. » (Bouthier, 2005). De fait, la technologie est un moyen par lequel les projets d'intervention peuvent se légitimer. De son côté, l'intervention vue sous l'angle d'un processus consistant à explorer, expérimenter, enseigner, évaluer les moyens de modéliser la complexité inhérente aux APSA, nécessite un usage rationnel des techniques ne serait-ce que pour structurer sa propre démarche. II s'agit donc d'aborder les techniques en prenant en compte leur développement historique. Pourquoi et comment ont-elles évolué ? Les réponses à cette question nécessitent une analyse anthropologique 


\section{eJRIEPS 23 avril 2011}

des pratiques sportives socialement reconnues. Pour prendre l'exemple des sports collectifs, Deleplace (1979) montre dans sa modélisation du rugby que c'est en analysant le rapport d'opposition que l'on peut expliquer les choix, les évolutions et les adaptations des pratiquants. Enfin, il apparaît déterminant de comprendre les modes de transmission et d'assimilation des techniques dans la perspective d'une diffusion et d'un développement de ce patrimoine humain. Un tel objectif conduit à préciser notre définition du concept d'intervention. Pour nous, l'intervention relève de tout acte par lequel un intervenant transforme les compétences du pratiquant, compétences ancrées dans la discipline sportive, avec pour perspective l'optimisation de sa performance. La notion d'acte engage à anticiper. II s'agit en effet de coordonner et de planifier des actions dans le but d'atteindre un objectif identifié. L'usage du terme «intervenant » fait référence aux différents milieux d'expression des pratiques sociales. L'intervenant peut être un enseignant, un entraineur ou un animateur. Quel que soit son statut, il se doit de transformer c'est à dire, mettre en œuvre les conditions qui permettent de passer de l'interprétation à la transformation du monde. II ne se contente donc pas d'une observation béate mais se saisit de ces données pour faire évoluer son projet de transformation. En ce sens, sa mission consiste à faire évoluer les compétences du pratiquant ou du groupe de pratiquants. De plus, ces compétences sont ancrées dans la mesure où elles relèvent de la capacité à contrôler les évènements susceptibles de se produire dans un environnement respectueux de la complexité des rapports fondamentaux de l'activité considérée (ce qui écarte toute activité dont l'objectif n'est pas de travailler sur ce rapport fondamental). II nous semble donc indispensable de réaliser cette quête qui permet de mettre à jour l'essence, le rapport fondamental compris comme l'ensemble des « relations entre des termes essentiellement déterminés l'un par l'autre et par l'unité qu'ils constituent » (Sève, 1980, p.75). Et c'est semble-t-il dans une triple relation, aux adversaires, aux partenaires et au système de marque (spécificité des cibles) qu'il faut aller chercher pour identifier ce rapport dans les sports collectifs (Éloi et Uhllrich, 2001). Au travers de la mise en relation de ces différentes entités, il convient alors d'optimiser le niveau d'habileté atteint, c'est à dire, de rendre adéquates les potentialités des pratiquants à leur niveau de performance maximum. Autrement dit, il s'agit de créer les conditions qui permettent à chaque individu d'atteindre l'état de maîtrise qui leur est potentiellement accessible. Dans cette perspective, la démarche technologique devient, nous semble-t-il, un dispositif pertinent de développement des recherches en intervention. 


\section{eJRIEPS 23 avril 2011}

\section{Modélisation et démarche technologique}

2. 1. La modélisation des activités

Qu'est-ce que modéliser ? Modéliser conduit inévitablement à faire un lien entre l'analyse des pratiques sociales de référence et les moyens mis en œuvre pour les enseigner. Martinand (1994) remet en cause le modèle classique de transposition didactique qui entretient le mythe d'un passage automatique de «savoir savant » à «savoir à enseigner » pour aboutir à «savoir enseigné ». Sa critique porte notamment sur trois points. Tout d'abord, il fait référence à une conception intellectualiste conduisant à oublier le rapport physique au monde réel. Ensuite, il soulève le problème de la recevabilité du savoir savant qui est souvent posé de manière a priori comme « légitime et légitimant ». Enfin il relève l'inadéquation d'un modèle qui promeut les disciplines scolaires qui se construisent pour transmettre des pratiques «qui ne sont pas forcément des savoirs savants appliqués » (Martinand, 1994, p. 67). II défend que l'élaboration de tout modèle devrait avoir deux ambitions. Tout d’abord, «la spécificité, la complexité de l'activité de l'enseignement, sa "professionnalité" doivent pouvoir être prises en compte et analysées de façon plus profonde ». Ensuite, « le rapport de l'école aux domaines de référence non scolaires, où les acquis scolaires doivent pouvoir prendre du sens, doit être élucidé de façon plus radicale » (Martinand, 1994, p. 67). Ces ambitions conduisent à expliciter les distinctions entre «pratiques et disciplines » mais aussi entre « école et référence ». Ainsi, la démarche de modélisation se situe pleinement dans une perspective technologique car elle nécessite :

- de partir de l'activité pour retourner à l'activité

- de prendre en compte la complexité des activités réelles

- de produire de nouveaux concepts permettant de caractériser des situations typiques - de permettre d'énoncer des règles d'efficacité.

Se pose alors la question de savoir comment il est possible d'enseigner une activité sans s'appuyer sur une modélisation ? Pourtant, dans la littérature, les modélisations sont rarement explicitées. La forme de l'enseignement est beaucoup plus souvent abordée mais les fondements sur lesquels reposent les contenus proposés restent flous ou implicites. Nous nous efforcerons de montrer plus loin à quel point le contenu à transmettre nécessite une relation dialectique avec la forme même de l'enseignement qu'il requiert.

2. 2. Travail de modélisation et registre de technicité 


\section{eJRIEPS 23 avril 2011}

La technicité est la capacité à mobiliser de façon adéquate une technique. En ce sens, la technicité est l'expression du rapport dialectique « objet de l'action/action ». Combarnous distinguait « trois composantes premières de la technicité :

- une composante d'apparence philosophique, la rationalité technique,

- une composante d'apparence matérielle, l'emploi d'engins (outils, instruments, machines, équipements),

- une composante d'apparence sociologique, les spécialisations » (Combarnous, 1984, p. 70).

Nul doute qu'il a inspiré Martinand (1994) lorsque celui-ci a proposé le concept de registres de technicité. En effet, cette relation entre la finalité de l'action et la technique employée nécessite qu'un sens soit identifié par le sujet. Ainsi, la distinction des différents registres est utile pour caractériser les différentes technicités. Elle permet en retour de catégoriser les travaux en technologie des APSA. C'est la raison pour laquelle modélisation et registre de technicité entretiennent une relation dialectique inévitable. Car pour sortir de la technique « maudite » (Garrassino, 1983) il est nécessaire de la relier aux effets qu'elle est censée produire. Dans le cadre des sports collectifs et à propos du registre de lecture, la prise en compte du dispositif de l'adversaire par le pratiquant doit permettre de jouer dans le mouvement collectif engagé ou de le transformer par une prise d'initiative individuelle. La technique mobilisée dépasse donc la simple réalisation gestuelle. Et il semble bien que la composante principale de l'action nécessite la capacité à lire le jeu. C'est en ce sens qu'il est fait référence au registre de lecture. Dans le cadre du registre de maîtrise, la capacité à faire croire à l'adversaire que l'on va faire quelque chose pour, en réalité, réaliser autre chose est déterminante. La « manipulation », cette capacité à leurrer l'adversaire, doit donc prendre en compte le paraître et le réalisé. La technique déborde donc la simple gestuelle puisqu'elle doit incorporer la prédiction de la réaction de l'adversaire. C'est typiquement le cas lorsque le passeur de volley-ball «pompe » sa passe pour faire croire au contreur central adverse qu'il va donner le $1^{\text {er }}$ temps d'attaque. C'est également le cas lors d'un cadrage débordement où le joueur en possession du ballon oriente le défenseur sur une mauvaise trajectoire pour le prendre à contre-pied. Construire de telles habiletés nécessite d'avoir une vision structurée des différentes situations de jeu. Mais comment décrypter le jeu sans une modélisation sousjacente ? N'existe-t-il pas des modélisations tacites? La situation d'urgence condamne souvent l'intervenant à aller au-delà de ce qu'il sait. II doit construire son exercice et mettre en action son groupe. II est contraint « de "dire" et d' "expliquer" la technique, malgré ces zones d'ombre. L'obstacle tient au fait que le technicien doit "montrer", "justifier", 


\section{eJRIEPS 23 avril 2011}

"commenter", même s'il ne sait pas tout. II doit, quoi qu'il en soit, parler, convaincre. II doit exposer. D'où se paradoxe tenace : "tout" dire alors que "tout" n'est pas connu, expliquer, alors que tout n'est pas maîtrisé » (Vigarello, 1991, p. 147). Mais la force de l'habitude condamne ces raisonnements pragmatiques à devenir force de loi. Ces principes envahissent les terrains de sport sans que quiconque n'en connaisse les fondements ou les origines. Ainsi, pas un éducateur ne peut concevoir l'enseignement des sports collectifs sans qu'un apprentissage technique ne fasse l'objet d'une partie spécifique de l'entraînement. « L'originalité du discours technique est évidente, dans ce cas, par rapport à celui de la science : il lui faut aller au delà (ou en deçà) du savoir savant. Il lui faut même parfois ruser avec lui » (Vigarello, 1991, p. 147). II existe donc des modélisations contradictoires? Notre point de vue n'est pas de proscrire ces raisonnements pragmatiques (nous n'en avons ni l'intention, ni les moyens). Mais il nous semble que dans le cadre de la filière STAPS, la démarche technologique appliquée aux APSA pourraient se fixer comme objectif de développer de tels travaux. Les modélisations ainsi mise à jour devraient permettre d'une part, d'expliciter l'adéquation entre choix des techniques et moment de leur mobilisation et d'autre part, la nécessaire adaptation de l'organisation générale du geste à la situation particulière de jeu en termes d'intensité, de vitesse, de précision, etc. Nous avons précédemment décrit (Ulrich \& Éloi, dans cette revue) les quatre registres de technicité utilisés pour caractériser les recherches technologiques en APSA (Martinand, 1994 ; Durey \& Bouthier, 1994). Nous en faisons un bref rappel maintenant.

\section{2. 1. Les différents registres de technicité}

- Le registre de maîtrise : il prend en compte les états de contrôle des techniques. Les recherches ayant trait à ce domaine concernent donc autant, les techniques sportives que les techniques de transmission de ces techniques par les formateurs. Mais elles s'étendent aussi aux techniques utilisées par le formateur de formateurs pour enseigner à apprendre ces techniques.

- Le registre de lecture : il recouvre l'étude des modalités de prises d'informations des sujets qui sont au centre de la pratique. Il englobe aussi celle du formateur qui évalue les actions de ses pratiquants mais également l'appréciation qu'un formateur peut formuler au sujet d'un futur intervenant en formation.

- Le registre de transformation : il désigne les conditions et procédures d'évolution des savoirs techniques. II concerne les moyens que les joueurs mobilisent pour s'approprier les techniques nécessaires à leurs intentions tactico-techniques. II s'intéresse pareillement aux méthodes utilisées par le formateur pour inciter ces évolutions chez ses pratiquants. 


\section{eJRIEPS 23 avril 2011}

De la même façon, ce registre fait référence aux modalités retenues par le formateur de formateurs pour mettre en place un dispositif de construction de connaissances d'ordre didactique.

- Le registre de participation : il relève de l'analyse de l'activité déployée au delà de la pratique de la discipline sportive en elle-même. On envisage ici les nombreux rôles qui encadrent la pratique et la rendent possible. Les pratiquants peuvent devenir arbitre, manager ou observateur. L'intervenant éducatif peut être amené à pratiquer, à arbitrer, à organiser. Le formateur de formateurs est susceptible de mener des recherches, de préparer à divers concours, de dispenser des cours théoriques sur les pratiques.

\section{2. 2. Complémentarité des registres de technicité}

La nécessaire distinction des registres de technicité ne doit pas amener à envisager les contributions qui les alimentent comme des travaux cloisonnés et isolés les uns des autres. Cela est aussi vrai pour les activités concrètes développées par l'ensemble des acteurs qui interviennent dans les différents registres. Nous avons proposé de décrire ces travaux en spécifiant un registre principal et des registres associés. II paraît évident que les avancées dans un registre auront toujours des conséquences sur la problématisation des autres registres. Par ce travail imbriqué de modélisation de la pratique, de détermination des techniques et des technicités organisées selon les registres, les recherches technologiques jouent un rôle dans le développement de la culture des APSA. Reste à éclaircir la question de la transmission de cette culture à tous les échelons de la chaine de l'intervention éducative (pratiquants, intervenants, formateurs de formateurs). II est alors nécessaire d'envisager les moyens par lesquels ces nouvelles problématiques relatives à la technicité pourraient être partagées. Et c'est ici que la question des outils se pose de façon cruciale. Or, il se trouve que ces outils peuvent revêtir des formes extrêmement variées. A l'instar de différents auteurs (Cole, 1990, Norman, 1993 ; Rabardel, 1995; Vérillon, 2005) nous proposons de faire référence au concept « d'artefact». Comment positionner ce concept au regard des registres de technicité décrits plus avant?

\section{Les artefacts}

Qu'est-ce qu'un artefact? II est classiquement défini comme un objet plus ou moins complexe. A coup sûr, son existence contient l'empreinte de l'homme. Pour le définir, nous tenterons de dépasser les notions de phénomène, d'artifice, de gadget voire de parasite.

3. 1. L'artefact : alternative au concept d'objet technique 


\section{eJRIEPS 23 avril 2011}

Dès l'introduction de son ouvrage «Les hommes et les technologies », Rabardel (1995) nous rappelle que les objets et systèmes techniques sont improprement nommés. En effet, une telle dénomination pourrait laisser sous-entendre que ces dispositifs se génèrent de façon autonome. Or, il est patent que de leur origine à leur destruction (ou à leur recyclage), l'action de l'homme sur ces « outils » est permanente et cela aussi bien sur le versant de leur conception que sur celui de leur utilisation. Nous ne décrirons pas ici les raisons qui ont conduit à cheminer du terme « d'objet technique » à celui « d'artefact » (Rabardel et Vérillon, 1985 ; Rabardel, 1995). Disons que cette distinction marque le passage d'une vision technocentrique à une vision anthropocentrique. En effet, l'utilisation du concept « d'artefact » marque le basculement vers une conception qui accrédite l'idée que l'usage de ces outils contribue, dans un rapport dialectique inéluctable, à développer autant l'activité humaine que l'évolution de ses objets. «La notion d'artefact désigne en anthropologie toute chose ayant subi une transformation, même minime, d'origine humaine » (Rabardel, 1995, p. 59). L'intérêt d'une telle appellation est d'ouvrir le répertoire de ces artefacts aux objets non matériels. Et de fait, matériel ou non, la visée de ces constructions reste la même. Elle consiste à produire un effet propice. L'artefact contient donc en lui deux versants. D'une part, il est finalisé dans la mesure où il vise à apporter une solution avantageuse à un problème posé. C'est donc dans la recherche d'une issue favorable à une difficulté que se trouve l'origine des transformations (des adaptations) de l'objet initial. D'autre part, la mise à l'épreuve de ces artefacts ne présage pas des usages futurs qui pourront en être fait. Ces « inventions » sont donc socialement déterminées. En amont, puisqu'elles répondent aux besoins des pratiques réelles du secteur concerné. En aval, du fait qu'elles sont investies par le milieu social qui s'en empare et en fait parfois usage au delà des modalités initialement envisagées. «A chaque artefact correspondent des possibilités de transformations des objets de l'activité, qui ont été anticipées, délibérément recherchées et qui sont susceptibles de s'actualiser dans l'usage » (Rabardel, 1995, p. 60). Il est fait référence ici à une distinction primordiale entre l'artefact en tant qu'objet (matériel ou non) et l'appropriation de celui-ci par un acteur singulier dans un contexte particulier car c'est cette appropriation qui va marquer le passage de l'artefact à l'instrument.

\section{1. 1. De l'artefact à l'instrument}

En effet, cette «genèse instrumentale » requiert un double mouvement. Non seulement, l'appropriation de l'artefact par le sujet qui l'utilise. On parlera d'instrumentation. Mais également, la prise en compte des événements qui contribuent à l'évolution de l'artefact. On emploiera alors le terme d'instrumentalisation. Pour Rabardel, « les deux processus 


\section{eJRIEPS 23 avril 2011}

contribuent solidairement à l'émergence et l'évolution des instruments même si, selon les situations, l'un d'eux peut être plus développé, dominant, voire seul mis en œuvre » (Rabardel, 1995, p.138). L'unité instrumentale ne peut donc être saisie que dans la prise en compte de cette double orientation de l'activité du sujet. Trouche (2005) précise que « cette distinction est fondamentale :

- elle met en évidence que les artefacts ne sont que des propositions, qui seront développées, ou non, par un sujet ;

- elle met en évidence que ce développement se fait au cours d'un processus, la genèse instrumentale, où l'activité de l'usager et le contexte de cette activité sont décisifs ;

- elle met en évidence que tout instrument a une partie matérielle (c'est la part de l'artefact qui a été sollicitée au cours de l'activité) et une partie psychologique (c'est l'organisation de l'activité, dans un but donné, ce que Vergnaud, après Piaget, appelle les schèmes) 》 (Trouche, 2005, p. 272). Ainsi, la genèse instrumentale faire référence à une pluralité de transformations dont les finalités sont distinctes bien que complémentaires et qu'il nous faut préciser ici.

\section{1. 2. Instrumentation, instrumentalisation et catachrèse}

L'instrumentation désigne les processus orientés vers le sujet lui-même qui font références à la création et à l'évolution de schèmes relatifs à l'utilisation de l'artefact. L'artefact a donc une influence sur la manière dont le sujet va orienter son activité de résolution de problème. «L'instrumentation, c'est donc ce processus par lequel les contraintes et les potentialités d'un artefact vont conditionner durablement l'action d'un sujet pour résoudre un problème donné » (Trouche, 2005, p. 274).

L'instrumentalisation est dirigée vers l'artefact. Elle concerne la production et l'évolution des artefacts pouvant aboutir parfois à un usage catachrétique de ceux-ci. Ici, le pouvoir d'innovation du sujet s'exprime au travers d'une démarche de personnalisation de l'artefact afin de l'adapter à son propre usage. « Ce processus peut être considéré comme un détournement ou comme une contribution de l'usager au processus même de conception de l'instrument »(Trouche, 2005, p.275). La question peut être légitimement posée de savoir jusqu'à quel point le processus d'instrumentalisation ne nécessite pas d'une manière systématique le détournement ou au moins l'adaptation de l'artefact préconisé. Serait-il déraisonnable d'envisager que cette déviation par rapport à une utilisation classique de l'outil (telle que préconisé par le concepteur) soit nécessaire dans le but de le rendre fonctionnel pour le sujet ? La catachrèse n'est-elle pas l'ingrédient indispensable qui permet de transformer le dispositif proposé à tous en un instrument adapté à soi. Ne peut-on pas voir là un mode d'expression de la créativité humaine ? Mais 


\section{eJRIEPS 23 avril 2011}

si la catachrèse est un moyen détourné de réaliser l'irréalisable, il convient de noter que cela est certainement dû à l'impossibilité de concevoir des artefacts d'une portée universelle. L'usuel conflit qui met en scène la tâche, le sujet, voire les autres, ressemble à un piège duquel il faut bien réussir à se sortir. Pour le dire à la manière de Clot, «la catachrèse trahit ainsi l'irreprésentable ou l'irréalisable au moment même où elle l'efface en l'accomplissant, où elle le masque en le définissant. Paradoxalement, en figurant l'impossible et en lui offrant un autre destin possible que l'impasse, elle le développe » (Clot, 2003, p.17). Cette double orientation de la genèse instrumentale (instrumentation comme appropriation de l'artefact et instrumentalisation comme développement catachrétique de cet artefact) nous renvoie, dans notre domaine, à l'usage quasi systématique de situations d'apprentissage déviantes vis à vis de la situation de référence (la vraie situation de match, de course, de combat, etc.). Comment envisager l'enseignement des APSA sans qu'une transposition en termes de vitesse, d'intensité ou de contraintes ne soit organisée dans ces situations ? Jusqu'à quel degré d'adaptation peut-on aller sans dénaturer les conditions authentiques de la pratique ? De fait, la stratégie de l'intervenant consiste à introduire un décalage plus ou moins important (optimal !) entre les sollicitations de ces situations et les sollicitations de la situation réelle. Le but consiste à développer une adaptation mobilisable ultérieurement dans la situation authentique de production de la performance. II s'avère que l'un des procédés régulièrement mobilisés consiste à introduire un obstacle mineur mais réel dans l'activité des pratiquants. L'enjeu de cette entreprise est de focaliser leur attention sur un aspect jugé déterminant de l'activité. Cependant, ces mêmes situations n'ont de valeur qu'instrumentalisées par le pratiquant. Nous retrouvons ici l'idée de conceptualisation en acte développée par ailleurs (Vergnaud, 1996). En effet, le pratiquant va construire des principes organisateurs de l'action dans le cadre de la régulation de sa propre activité. La perspective catachrétique laisse alors à penser que les acteurs vont faire évoluer la situation initiale, processus permettant de les placer dans une dynamique de progrès. Une telle manœuvre relève bien, nous semble-t-il, de la genèse instrumentale. On perçoit à l'issue de cette description de l'artefact, l'importance que ce concept peut avoir dans les APSA aux trois niveaux de l'intervention: pratiquant, intervenant éducatif, formateur de formateurs.

\section{1. 3. L'incident comme artefact}

Nous l'avons indiqué plus haut, l'artefact peut revêtir des formes matérielles ou non. Dans le contexte des APSA, il s'avère que les intervenants sont amenés à employer au même titre des situations concrètes ou des images. La gestion du degré de distorsion des 


\section{eJRIEPS 23 avril 2011}

situations d'entraînement comme des représentations de l'action est donc un élément de l'activité professionnelle de l'intervenant. Elle contribue à décrire ou à faire évoluer la pratique. II n'est donc pas rare que dans ces situations d'apprentissage ou d'entraînement de tels incidents soient utilisés comme leviers de développement du pratiquant. Nous proposons donc d'étendre le concept d'artefact à ces incidents volontairement introduits dans l'activité du sujet en vue de transformer son expérience. Cette extension nous est apparue nécessaire pour préciser l'arsenal artefactuel utilisé par l'intervenant en APSA dans son projet d'intervention. À quoi faisons-nous allusion lorsque nous parlons d'incident ? L'utilisation du concept d'incident se trouve nous semble-t-il justifiée dans la mesure où il est fait référence à un événement qui n'entrave pas l'activité majeure des personnes impliquées. II s'agit d'une difficulté secondaire qui « survient » dans le cours d'une action principale. Dans notre domaine, cet événement, fortuit pour le sujet mais volontairement introduit par l'intervenant, apparaît dans le cadre de la pratique avec pour but de poser une difficulté réelle, inopinée et ciblée aux protagonistes. L'insertion de cet événement a pour but de provoquer une compréhension nouvelle de la situation qui engage à produire une adaptation. Adaptation dont on souhaite qu'elle soit à nouveau mobilisable dans la situation réelle (réinvestissement possible d'une situation d'entraînement dans une situation de référence). On pourrait faire un parallèle avec la définition grammaticale d'une proposition incidente dont on dit qu'elle est insérée dans une autre, afin d'apporter une précision de sens supplémentaire. L'incident artefactuel peut donc être caractérisé comme l'utilisation d'un objet matériel ou non, volontairement introduit par l'intervenant dans l'activité du sujet visant à la modification du cours normal de sa pratique dans le but de provoquer une compréhension nouvelle de son activité. L'instrumentation de ce type d'artefact reste évidemment soumise à l'usage qu'il en sera fait par le pratiquant.

\section{2. Les différentes formes artefactuelles}

Comme nous l'avons montré plus haut, la genèse instrumentale engendre des processus d'émergence et d'évolution des instruments. II serait d'ailleurs plus judicieux d'évoquer, comme le propose Rabardel et Bourmaud (2009), des systèmes d'instruments. En effet, les instruments ne sont pas isolés les uns des autres et c'est en fonction des sollicitations de la situation particulière qu'un ensemble spécifique d'instruments est constitué. « C'est la logique de notre activité située concrète et singulière qui, dans ce cas, a organisé les relations de complémentarité fonctionnelle entre les instruments et les séquences temporelles de leurs usages successifs ou concomitants » (Rabardel et Bourmaud, 2009, p.211). En fonction des contextes concernés, les systèmes d'instruments développés par 


\section{eJRIEPS 23 avril 2011}

les sujets organisent donc une multitude d'artefacts. De ce fait, les classes d'artefacts peuvent être diverses : matériel, sémiotique, symbolique... Dans le contexte des APSA, nous proposons de recourir à quatre classes d'artefacts qui semblent pertinentes pour couvrir le champ de l'intervention en STAPS.

\section{2. 1. Des formes artefactuelles déjà référencées}

II a déjà été distingué trois formes d'artefacts pour spécifier l'intervention en APSA. « La technologie, au-delà des réalisations matérielles sophistiquées, est considérée comme une science humaine de l'intelligence mise jeu dans la conception, la mise au point et l'évaluation de créations, une science de l'artificiel générant des artéfacts cognitifs, matériels, et bien sûr corporels » (Bouthier, 2008 a, p. 47). Nous nous proposons de rappeler maintenant la nature de ces différents artefacts.

- Les artefacts cognitifs qui sont des éléments artificiels et conceptuels conçus pour traiter l'information dans le but de satisfaire une fonction représentationnelle. (Cole, 1990, Norman, 1993). Ils tendent à influencer les représentations construites à propos de l'activité. Par exemple en sports collectifs, le concept de rapport d'opposition attaque/défense ou bien celui de point de fixation. II s'agit ici de concepts abstraits qui peuvent s'actualiser au travers d'indicateurs observables. Cette idée se rapproche de la notion de concept pragmatique telle qu'elle est développée par Pastré.

- Les artefacts corporels qui interviennent lorsque le sujet mobilise des éléments d'actions tactico-techniques en adéquation avec une perception de la situation. II peut s'agir d'une reconnaissance in situ de la forme de jeu intégrée auparavant (à l'entraînement) dans le moment où elle se produit (Uhlrich \& Bouthier, 2008). Par exemple, au rugby, « ressentir » la prise d'intervalle au moment du franchissement de la ligne de front. Au volley-ball, pour le passeur, «voir» le contreur central adverse sauter sur le $1^{\text {er }}$ temps et donner la balle à l'attaquant de $2^{\mathrm{e}}$ temps (Éloi, 2009). Ces deux exemples imposent d'avoir mis en adéquation les informations provenant des différents canaux sensoriels et une prise de décision concomitante de l'engagement physique qui en résulte. L'artefact mobilisé est corporel en ce sens qu'il fait appel à tous les éléments constituants de la situation présente mis en relations avec les données engrangées soit sous forme de modèles, soit sous formes de situations repérées auparavant. Les vitesses de déplacement, les intervalles mobilisés, les positions et les prises de décisions des adversaires sont autant de facteurs qu'il faut organiser pour se guider dans l'action. C'est l'outillage corporel qui permet d'exécuter une telle tâche que nous appelons artefact corporel. 


\section{eJRIEPS 23 avril 2011}

- Les artefacts matériels qui donnent de nouveaux pouvoirs aux utilisateurs. Ils permettent aux sujets de disposer des moyens d'orienter et de réaliser l'activité (Rabardel 1993 ; Vérillon, 2005). C’est un outil technique qui est chargé de conceptualisation. En effet, l'élaboration de l'outil a nécessité de rationaliser les différentes formes de pensées relatives à la technique, la tactique, la stratégie, ainsi qu'au développement des modèles de l'activité. De ce fait, il dépasse un simple aménagement du milieu. C'est par exemple en sport collectif :

- la grille d'observation : les critères d'observation sont le résultat d'une pensée en acte sur l'activité. Ils font référence à une modélisation (quel que soit son degré d'explicitation). Cette pensée se décline alors dans les interprétations comme dans les interventions qui suivront.

- le dispositif de la situation : l'organisation de la situation implique une conceptualisation des modes de réalisation en lien avec la modélisation de l'activité.

- l'outil vidéo dans une perspective d'analyse du jeu : l'organisation de la prise de vue est en relation directe avec la conception de la pratique. Si l'on a compris que l'espace de jeu est orienté par deux cibles (Deleplace, 1979) alors, il apparaît nécessaire que la prise de vue soit placée sur cet axe pour documenter les actions décisives.

II reste qu'une autre forme d'incidents est souvent utilisée à des fins de formation. Nous voulons parler maintenant des adaptations réglementaires particulièrement usitées dans l'enseignement des sports collectifs.

3. 2. 2. Une nouvelle forme d'artefacts : les artefacts réglementaires

La fréquence de l'utilisation des adaptations réglementaires dans le domaine des APSA (plus particulièrement dans les activités d'opposition) nous semble justifier l'intronisation d'une nouvelle forme d'artefacts typiques de l'intervention en APSA. Ainsi, nous avons voulu compléter cette caractérisation en introduisant une quatrième catégorie: les « artéfacts réglementaires».

La mise en place de la règle aboutit à toute sorte d'interprétations. Elle fait l'objet de débats. Elle se retrouve au centre de polémiques. La règle est donc vivante. La règle organise la rencontre. Elle contraint l'action des protagonistes. Elle définit le champ des possibles (Éloi, dans cette revue). De ce fait, elle provoque des incidents dans la production corporelle. Elle contribue dans le même temps à contenir la pratique tout en la développant. II semble donc opportun d'identifier, selon les cas, deux effets à l'utilisation de la règle :

- $1^{\mathrm{er}}$ cas de figure : la règle génère en elle-même de l'incident. 


\section{eJRIEPS 23 avril 2011}

Ainsi, pour le néophyte, la règle du « tenu » en rugby organise le rapport plaqueur/plaqué dans un souci de continuité du jeu. Cependant, lâcher le ballon et le laisser libre n'est pas naturel pour le joueur impliqué dans l'action. II doit donc s'affranchir de la tentation de le conserver. La mise en place de cette règle de façon précoce, dans la Période Initiale d'Appropriation du Jeu (Éloi \& Ulrich, 2001) rentre en contradiction avec la libre manipulation du ballon. Ici, la règle implique une organisation corporelle nouvelle dans la mesure où le joueur doit apprendre à tomber orienté vers son camp afin de libérer le ballon vers ses partenaires. Dans ce premier exemple, l'application de la règle elle-même crée de l'incident et nécessite de l'adaptation.

- $2^{\mathrm{e}}$ cas de figure : la règle peut être momentanément modifiée pour provoquer une expérience particulière.

En volley-ball, les formes de jeu sont souvent favorisées (il «faut» faire 3 passes) au détriment $\mathrm{du}$ fond (la prise en compte des placements et des déplacements des adversaires). En supprimant temporairement la possibilité de faire des passes à son partenaire (en situation de 2 contre 2 et avec obligation de jouer à tour de rôle dans chaque doublette), on limite le champ des préoccupations. Ainsi, on oriente sciemment les pratiquants vers la prise en compte des antagonistes. II est probable que dans un premier temps, le débutant restera focalisé sur la trajectoire de la balle qui lui parvient pour assurer sa frappe de balle. Mais petit à petit, le jeu en renvoi direct va faciliter la prise en compte de l'opposition. À l'usage des différents faits de jeu qui vont apparaître de façon aléatoire, il va pouvoir identifier des classes de situations plus ou moins favorables. II lui sera alors possible de reconnaître celles dans lesquelles il pourra se focaliser sur les placements et déplacements de l'équipe adverse (quel est le joueur qui ne peut pas intervenir sur le renvoi à ce moment de l'échange). Dans ce second exemple, c'est la conception de la situation qui vise à créer l'incident, « là où un aménagement matériel impose une simple contrainte à l'activité de l'individu, l'artefact favorise une conceptualisation dans l'action » (Uhlrich \& Bouthier, 2008, p. 211). Dans le cas de figure du rugby comme dans celui du volley-ball, l'artefact réglementaire constitue un levier qui rend possible la compréhension du rapport d'opposition.

3. 3. Théorie de l'activité et articulation entre registres de technicité et artefacts La recherche technologique contribue à alimenter les différents registres identifiés. Dans ce contexte, les artefacts participent à deux niveaux. D'une part, ils permettent de mettre en perspective les données qui contribuent à élaborer la modélisation des APSA. Dans ce sens, les artefacts jouent le rôle de révélateur des logiques profondes des diverses disciplines. D'autre part, ils concourent à faire évoluer le niveau des pratiques. Ici, les 


\section{eJRIEPS 23 avril 2011}

artefacts permettent l'assimilation des techniques appropriées. II semble donc nécessaire d'envisager que la recherche technologique en STAPS alimente deux secteurs. Un premier secteur investi par les travaux de modélisation des pratiques sociales de référence (Martinand, 1986). Un second secteur qui s'oriente vers l'étude des moyens de transmission des techniques mobilisées dans les APSA. Le positionnement des registres et des artefacts dans un schéma général prend en compte le principe d'une valence modélisation et d'une valence ingénierie de formation. Cependant, c'est l'activité du sujet qui donne vie à l'artefact initial et l'engage dans un processus de développement durant lequel il prend alternativement le statut d'artefact et d'instrument. Une telle conception trouve une légitimité théorique dans les travaux issus du cadre d'analyse de l'activité telle qu'il a été proposé par la psychologie soviétique. «Dans le flux général de l'activité, Léontiev (1974) distingue des activités diverses caractérisées par leur motif, des actions orientées par un but et des opérations qui dépendent des conditions dans lesquelles le but doit être atteint » (Savoyant, $\left.{ }^{1} 1979-2010\right)$. Si la description d'un modèle général de l'activité conduit à séparer ses divers éléments, il faut préciser qu'un tel cadre d'analyse suppose que ces éléments sont en perpétuelle interrelation. II ne s'agit donc pas de se référer à une conception figée de ce cadre d'analyse qui envisagerait différents niveaux indépendants les uns des autres. La pertinence d'une telle théorie provient justement du fait qu'elle incorpore la dynamique des influences réciproques de chacun de ces éléments. Ainsi dans le lien activité-action, il est envisagé qu'en fonction des circonstances, l'activité puisse prendre le statut d'action et réciproquement. «Ainsi, si l'objet qui occupe la place de motif perd son caractère de stimulation il ne sera alors plus porteur que d'orientation et il occupera la place de but : l'activité définie par ce motif se transformera alors en action orientée vers ce but...» (Savoyant, 2010, p. 95). Au niveau de la relation actionsopérations, un tel processus est également identifiable. Ainsi, les actions formées «se transforment en opérations dans la mesure où elles sont incluses dans d'autres actions ayant une structure opérationnelle plus complexe, c'est à dire dans la mesure où elles deviendront les moyens d'exécution de ces nouvelles actions » (Savoyant, 2010, p. 96). Cette mutation du statut d'action vers celui d'opération s'explique par le fait que cette dernière quitte le domaine des processus conscientisés. II nous apparaît qu'un tel modèle théorique qui prend en compte que l'activité du sujet conduit à une alternance du statut d'action et d'opération n'est pas sans lien avec l'alternance des modalités de transformation de l'artefact en instrument et réciproquement. Ce point de vue nous semble

\footnotetext{
${ }^{1} 1979$ correspond au texte paru pour la première fois dans les Cahiers de psychologie, 22, p. 17-28.
} 


\section{eJRIEPS 23 avril 2011}

constituer une piste intéressante d'investigation pour de futurs travaux de recherche. Cette voie de réflexion pourrait corroborer l'hypothèse selon laquelle, c'est par cette alternance que se transmettent et se développent les savoirs techniques. Ainsi, cette relation de réciprocité entre artefact et registres de technicité nous semble s'exprimer au travers de trois modes de relations cycliques.

1. Les registres de technicité sont conçus comme des entités qui naissent des besoins liés à la pratique de terrain.

2. Les artefacts prennent vie dans le processus d'instrumentation.

3. Les instruments alimentent alors à nouveaux les registres

II est envisageable que les influences réciproques des artefacts et des registres expliquent d'une part, l'évolution des techniques humaines (au sens large) et d'autre part, la possible potentialisation des savoirs techniques.

\section{René Deleplace : un précurseur de la démarche technologique}

Comme nous l'avons proposé précédemment, les recherches en technologie des APSA peuvent être caractérisées par :

- la référence à des registres de technicité (principal et associés)

- l'usage de formes artefactuelles (matérielle ou symbolique)

- l'alternance du statut de l'outil d'artefact à celui d'instrument (et réciproquement).

Pour illustrer notre point de vue, nous prendrons quelques exemples parmi les travaux de René Deleplace ainsi que les prolongements qui ont pu en être fait. Pour saisir l'influence de cet auteur sur les travaux en intervention qui se développent aujourd'hui et pour comprendre la genèse de ce courant de pensée nous renvoyons le lecteur à l'hommage rendu par Bouthier à l'occasion du colloque de l'ARIS 2006 de Besançon (Bouthier, 2008 b).

\section{1. La double orientation des travaux de René Deleplace}

Dès les années 60, Deleplace (1966) pressent qu'il existe une alternative à la vision techniciste qui est alors à l'œuvre dans l'enseignement des sports collectifs. Cependant, cette alternative reste à construire. C'est dans «Rugby de mouvement, rugby total » (1979), que l'auteur développe totalement sa théorie du rugby qui emprunte deux voies parallèles bien qu'indissociables. D'une part, une analyse extrêmement précise de la complexité du jeu qu'il est à même d'organiser, de structurer et de systématiser et qui aboutit à une véritable modélisation du rugby. D’autre part, une logique de construction des situations d'apprentissage qui prend en compte les joueurs de tous les niveaux dans le respect de cette complexité. II nous semble que la pertinence de ses propositions tient à 


\section{eJRIEPS 23 avril 2011}

la force du lien dialectique qui unit les deux plans. Car c'est en partant de l'analyse du jeu qu'il va développer des propositions de construction des situations d'apprentissages qui sont encore novatrices aujourd'hui. En effet, cette méthode fait la démonstration qu'il est possible de développer les compétences du pratiquant en le confrontant à son adversaire. Elle postule que dès la première initiation, c'est dans la réalité du rapport d'opposition que peuvent se construire des apprentissages efficients. II engage un renversement radical d'une conception dont le mot d'ordre était « la technique première et la tactique peut-être » à une conception qui revendique « la tactique première et la technique avec ». Aussi, cette révolution de la manière de penser l'enseignement des sports collectifs requiert de développer un solide argumentaire. À l'origine de son travail se situe donc une astuce. C'est le statut d'une telle astuce que nous allons analyser maintenant.

4. 2. Une « astuce primitive » pour dévoiler «l'essence » du jeu

Pour mettre à jour les éléments qui engendrent la dynamique du jeu, nous sommes amenés à utiliser une « astuce primitive » (Deleplace, 1983). Ces mots très simples pour expliquer une intuition subtile nécessitent quelques explications. Car l'astuce qui a été révélée, bien qu'étant primitive, est loin d'être simpliste ou frustre. Au contraire, elle s'avère première, essentielle. C'est-à-dire que le terme «primitif » n'est pas à prendre dans son sens commun d'archaïque mais bien plutôt dans son sens philosophique de premier, d'essentiel. Cette astuce joue ici le rôle d'un artefact cognitif, c'est à dire un élément conceptuel conçu pour satisfaire une fonction représentationnelle. Un outil qui permet de créer un nouvel effet : mettre à jour la logique du jeu. Mais de quelle astuce s'agit-il ?

\section{2. 1. L'astuce primitive}

Le constat trivial que l'on peut faire au sujet des sports collectifs est relatif à la nécessaire présence : - de cibles (les buts, paniers, poteaux, ...)

- de projectiles (ballons).

Pourquoi d'autres activités nécessitant des cibles et des projectiles sont-elles fondamentalement différentes ? C'est en examinant les termes du problème et en cheminant vers la réponse à cette question que l'on va mettre à jour l'essence de la pratique, c'est-à-dire son rapport fondamental. Comparons le golf et le rugby.

- Au golf: chaque joueur a son projectile (sa balle). II y a donc plusieurs projectiles. De ce fait, les protagonistes utilisent tour à tour une seule cible.

- Au rugby : un seul projectile (une balle) pour 2 équipes. Plusieurs cibles : chaque groupe de protagonistes a sa cible. 


\section{eJRIEPS 23 avril 2011}

Chacune des mises en scène a comme conséquence de créer des rapports singuliers. La nécessité d'être en possession du projectile pour atteindre la cible adverse structure le jeu de rugby en mettant en avant que c'est la lutte pour entrer en possession du projectile qui est première. Au golf, chacun peut prendre son temps pour ajuster son coup dans le silence respectueux de la concentration de l'adversaire. Au rugby, la lutte pour maîtriser, y compris au moyen de sa force physique, l'adversaire porteur du ballon organise une pression spatio-temporelle de tous les moments. Au golf, l'atmosphère feutrée (et parfois humide) des greens, où chacun use de courtoisie pour se mettre en conformité avec « l'étiquette ». Au rugby, moins de politesse. Si je suis plus fort, je pousse. Si je trouve l'intervalle, je m'engouffre.

Le statut de l'artefact cognitif développé ici pour dévoiler la nature du jeu alimente consécutivement le registre de transformation. En effet, l'objectif de cette astuce est de dévoiler aux pratiquants autant qu'aux autres intervenants (les collègues) une nouvelle voie à emprunter. Cet artefact cognitif tente donc de faire évoluer les procédures d'apprentissage des savoirs techniques. II a pour objectif d'influencer les méthodes utilisées par les formateurs pour transformer les pratiques.

\section{2. 2. L'essence du jeu}

Cette astuce «primitive » dévoile donc plus que la simple forme visible du jeu. Elle met en évidence son essence c'est-à-dire, du point de vue du matérialisme dialectique, le rapport fondamental de l'activité. Elle dévoile que c'est le rapport à l'adversaire qui est essentiel. Ainsi, dans les phases à l'initiative des opposants, la lecture du jeu adverse permet l'anticipation et le blocage de ses initiatives offensives. De même, dans les phases à l'initiative de notre équipe la prise en compte du dispositif défensif adverse guide les choix au sein des différentes alternatives d'attaques possibles. Le combat pour contrôler le projectile n'est donc pas un combat comparable à celui du boxeur qui à chaque coup porté marque des points. Ici, le combat qui est livré n'est que le moyen de rentrer en possession de l'objet par l'intermédiaire duquel il sera possible de marquer des points. Le rugby n'est donc pas à classer parmi les sports de combat même si l'engagement y est parfois considérable. Le combat ne constitue pas le but des protagonistes mais le moyen de conserver ou de récupérer le projectile. Je ne marque aucun point même si mon «plaquage » fait perdre le ballon à mon adversaire. Je ne marque pas plus de point si mon « raffut » laisse à terre mon défenseur. Pour marquer des points, je dois atteindre le système de marque, c'est-à-dire que je dois atteindre à l'aide du projectile une cible réglementairement définie. Et dans les sports collectifs, les cibles ne sont jamais les protagonistes. 


\section{eJRIEPS 23 avril 2011}

\section{2. 3. D'un artefact à l'autre}

On peut suivre ici l'itinéraire de la pensée Deleplacienne. Sa pratique de formateur engendre une incompréhension. II s'agit d'abord de mettre en évidence le hiatus pour luimême. Mais il faut aussi en faire la démonstration devant les collègues. II faut convaincre les pairs. La matérialisation du décalage entre l'essence de l'activité pratiquée et la méthode avec laquelle elle est enseignée aboutit à l'inéquation mathématique suivante :

«Un seul projectile et deux cibles» est différent de «plusieurs projectile et une seule cible ».

Les méthodes d'enseignement ne peuvent donc être similaires. La pensée initiale qui aboutit à cette démonstration trouve une seconde vie lorsqu'elle doit être partagée. Ce cheminement illustre comment cet artefact cognitif à l'origine, lorsqu'il est employé pour comprendre la réalité de la pratique, devient un instrument de conception pour celui qui s'en empare et se transforme ensuite en un artefact matériel lorsqu'il est utilisé pour démontrer aux autres la validité de la démonstration. En effet, cette inéquation une fois posée sur le papier permet d'orienter l'activité de compréhension de ceux qui partage les mêmes préoccupations. Elle est un outil chargé de conceptualisation. Pour en arriver à cette apparente limpidité, il a fallu rationaliser la pensée relative à la technique, la tactique ou la stratégie. In fine, un tel artefact a pour ambition de permettre aux autres de disposer des moyens d'orienter et de réaliser leur activité (qu'ils soient intervenants ou pratiquants). Notre conception de la technologie des APSA est anthropocentrée dans ce sens qu'elle permet de comprendre la technique dans sa forme évolutive et non pas comme produit donné tel quel. Nous avons déjà considéré (Ulrich, Eloi \& Bouthier, dans cette revue) la technique comme contextualisée selon ses origines, son environnement et ses finalités. Nous savons par ailleurs que cette technique est évolutive, qu'elle subit les influences qui proviennent de ses usages, de son contexte géographique ou encore des contraintes réglementaires qui infléchissent sa mobilisation. Se pose alors la question de sa transmission. C'est dans cette dynamique que l'artefact matériel prend toute sa consistance. En orientant l'activité de l'individu (pratiquant, intervenant éducatif ou formateur), il participe à la construction d'une véritable base d'orientation de l'action (Galpérine, 1966).

Ainsi, développant son analyse, Deleplace est conduit à construire un artefact matériel qui prend la forme d'une représentation graphique de « l'astuce primitive ». Bouthier relève que « au delà du rugby, Deleplace apporte une contribution cruciale à la modélisation, l'enseignement et l'entraînement des sports collectifs » et notamment, « il approfondit le concept d'unité dialectique des rapports d'opposition attaque <=> défense ». (Bouthier, 


\section{eJRIEPS 23 avril 2011}

2008 b, p. 47). Il en vient alors à poser que pour tout sport collectif : noyau primitif du jeu = effets d'opposition. On peut donc immédiatement symboliser le dynamisme logique fondamental du jeu dans le schéma suivant (Deleplace, 1983, p.6).

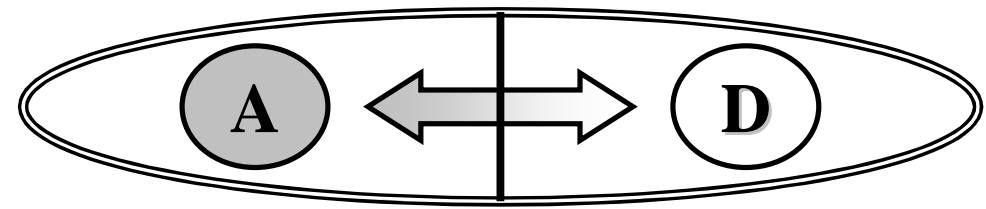

A A figure l'antagoniste possédant la balle à l'instant donné.

(D) D figure l'antagoniste ne possédant pas la balle dans ce même instant

La double flèche signifie que dans cet instant, l'acte de A n'a de sens que rapporté à celui de D au même instant et vice et versa.

L'enveloppe symbolise l'unité indestructible de l'acte complexe à tout instant [A en opposition à $\mathrm{D}, \mathrm{D}$ en opposition à $\mathrm{A}]$.

La ligne verticale coupant la double flèche en deux et séparant en deux l'enveloppe symbolise le caractère à tout instant antagonique de l'acte complexe total unique.

Figure 2. Symbolisation de la logique du jeu (Deleplace, 1983, p.6)

II devient alors possible de s'appuyer sur l'artefact matériel pour provoquer la transformation des représentations initiales chez les autres (collègues, étudiants, élèves). La vie de l'astuce initiale se trouve alors prolongée par les usages futurs qui en seront faits.

4 .3. Du rugby aux sports collectifs : une seconde vie pour l'astuce primitive La question légitime que nous devons nous poser concerne la possible extension d'une telle analyse aux autres sports collectifs. Les travaux de René Deleplace nous permettentils d'avancer sur la voie de l'élaboration d'une réponse à la question de l'essence des sports collectifs ? Le rapport fondamental y est-il identique ? II nous semble bien que l'astuce primitive développée pour dévoiler l'essence du rugby tient toutes ses promesses lorsqu'elle est appliquée aux autres sports collectifs. Nous devons, à ce point de l'exposé, préciser la nature des activités physiques auxquelles nous faisons référence. Ce que nous appelons sports collectifs dans cet article recouvre les activités collectives d'opposition dont les espaces de jeu sont orientés par deux cibles situées aux extrémités de cet espace. Cette organisation permet notamment la réversibilité possible à tout moment des rôles d'attaquants et de défenseur ainsi que la possibilité pour les protagonistes de se 


\section{eJRIEPS 23 avril 2011}

transmettre l'unique projectile mis à la disposition des deux équipes. II ne sera donc ici question ni de double au tennis, ni de base-ball. Pour une analyse plus détaillée, le lecteur pourra se reporter à une précédente présentation (Éloi \& Ulrich, 2001). Ainsi, nous pouvons constater que pour tous les sports collectifs, la mise en scène est similaire :

- Deux équipes qui tentent d'atteindre la cible adverse en même temps qu'ils protègent la leur.

- Un seul projectile disponible pour les deux équipes.

De cette disposition particulière nait la contradiction. En effet, l'unicité du projectile a comme conséquence que sa possession par une équipe se fait au détriment de l'autre équipe. Et il semble bien que ce « ressort » du jeu fonctionne de la même façon dans des activités aussi différentes que le hand-ball, le football ou le volley-ball (pour ne citer que celles-là).

4. 3. 1 Sur la symétrie des espaces de jeu

Pour Bernard Jeu, «le sport emprunte les voies que lui suggèrent les rituels anciens ». Les raisons qui font que telle activité a un attrait important pour les humains peuvent avoir des racines profondes. «Ainsi on joue avec le jeu et, en opposant la structure du sacrifice à elle-même par l'effet de la symétrie, on dissimule ce que l'on simule et on ne sait plus qui sera le sacrifié ni qui sera le sacrificateur. II apparaît simplement qu'on ne veut pas perdre et que l'autre est décidé à gagner » (Jeu, 1983, p.253). On peut en effet s'interroger sur l'intérêt suscité par les sports collectifs. Par le fait qu'ils sont nés ensemble (fin du $19^{\mathrm{e}}$ siècle) et qu'ils ne cessent de se développer. Jouer sa vie dans une épopée fictive (le championnat) sans jamais risquer de la perdre peut s'avérer être une aventure humaine contemporaine des plus excitante. Le rite sacrificiel réitéré génération après génération trouverait ici une actualisation moins barbare que par le passé (les sacrifices humains). La structure de la symétrie offrant par ailleurs autant de chance à l'un comme à l'autre par l'alternance des rôles de sacrifiés et de sacrificateurs.

\section{3. 2. Sur l'unicité du projectile}

On le comprend aisément maintenant. C'est l'organisation de l'espace de jeu liée à la mise à disposition d'un projectile unique qui structure la rencontre. L'organisation spatiale marque l'inéluctabilité de l'affrontement en même temps que l'unicité du projectile a deux conséquences déterminantes. La première fixe le rapport à l'adversaire. Nulle échappatoire possible. Si je veux marquer, je dois me saisir du projectile. Le combat est donc inévitable. II nécessite de rompre les velléités offensives des adversaires. Quel meilleur moyen que de lui retirer l'usage du projectile? La seconde fixe le rapport aux partenaires. La dimension collective de l'affrontement met en avant que certaines 


\section{eJRIEPS 23 avril 2011}

solidarités sont possibles. L'unicité du projectile nécessite donc de mettre en œuvre des coopérations permettant de déjouer les manœuvres défensives de l'adversaire. II s'agit d'assurer la continuité du mouvement offensif qui a comme seule fin l'atteinte du but adverse. Ainsi, dans ce dispositif d'une simplicité déconcertante s'enchevêtrent continuité et rupture en même temps que se nouent dans l'opposition de véritables enjeux de coopération. Fallait-il encore que Deleplace nous dévoile une telle logique ? II aura tout au long de sa vie développé des idées fondamentales pour la recherche en STAPS, cherchant inlassablement à «étudier l'intervention des hommes de terrain pour « transmettre le vécu perceptif corporel », en jouant en aller-retour sur le couple : « aide à la domination effective de la partie consciente sur la partie inconsciente » et «à la confrontation du sujet avec une activité physique consistante » » (Bouthier, 2008 b, p 48).

\section{Conclusion}

Si la technologie est une science humaine des techniques (Haudricourt, 1987), il reste que dans le champ des STAPS ce concept est particulièrement composite. Comme le note Bouthier, différentes périodes peuvent être identifiées quant au développement des travaux dans ce domaine. Nous adhérons au constat que la perspective la plus fructueuse à l'heure actuelle repose sur une conception « anthropo-techno-didactique », approche qui consiste à recontextualiser les techniques et «à les étudier comme un contenu culturel orienté vers la transmission » (Bouthier, 2008 a, p.49). Le moment ne semble pas encore venu de donner une définition achevée de la «Technologie des APSA ». II nous paraît plus approprié de décrire ce qui relève d'une «démarche technologique » pour la recherche en STAPS. Cette démarche tient en quatre points.

\section{1. Une visée}

En récupérant des données sur le terrain dans la situation réelle de l'action sportive, l'objectif est de créer les conditions d'une proposition utile pour les pratiquants ou les intervenants. II s'agit donc de ne pas en rester au plan des conclusions scientifiques. Les concepts scientifiques sont d'ailleurs enchâssés dans la réalité de la pratique sportive comme dans celle de l'intervention éducative en sport. On pourrait alors se poser la question de la validité de concepts «purement» scientifique en STAPS. Dans cette logique, les recherches technologiques prennent inévitablement en compte la complexité de l'action sportive. L'une des principales conséquences de cette perspective est qu'il faut accepter qu'il n'y ait pas de réponse définitive à une problématique soulevée. «Ce phénomène est complexe précisément parce qu'il tient pour certain l'imprévisibilité potentielle des comportements : il ne postule pas un déterminisme latent qui permettrait à 


\section{eJRIEPS 23 avril 2011}

une « intelligence assez puissante » de prédire par le calcul l'avenir de ce phénomène, fut-ce en probabilité » (Lemoigne, 1999, p.3).

5. 2. Une méthode : l'imbrication de registres de technicités et d'artefacts Au-delà de la connaissance des processus, la démarche technologique cherche à valider des résultats par la proposition d'essais techniques. II n'y a donc pas d'usage normalisé. L'étude se construit en fonction de la récupération des données utiles pour l'intervention. Malgré cette incertitude, la recherche en technologie des APSA interroge les registres de technicité (maîtrise, lecture, transformation ou participation) dans leurs interactions. Aussi nous semble-t-il judicieux de caractériser les travaux en spécifiant un registre principal et un ou plusieurs registres associés. II s'agit alors de mettre en évidence la nature des relations qui les lie. Dans ce cadre, la mobilisation d'artefacts constitue le moyen par lequel ces registres sont sollicités, développés et adaptés. II est alors nécessaire d'alimenter les dispositifs de formation (des pratiquants ou des futurs intervenants éducatifs) en créant des incidents artefactuels de natures différentes afin que les situations «formelles » d'enseignement cèdent progressivement la place à des situations plus fonctionnelles (Pastré, 1999 ; Samurçay \& Pastré, 1995).

\section{3. Des connaissances établies sur un objet technique}

Les recherches technologiques visent à élargir les procédures d'action des pratiquants et des intervenants éducatifs en envisageant à tous les niveaux une évolution des techniques. Qu'il s'agisse de la pratique ou de l'intervention éducative, voire de l'imbrication des deux, la mobilisation des résultats de ces recherches permet aux acteurs de réaliser une action conceptualisée qui oriente en retour une conceptualisation en acte (Vergnaud, 1996). Nous atteignons alors le niveau de la technicité par :

- L'expression de problématiques, concepts et normes.

- La mobilisation d'outils matériels ou symboliques.

- L'installation du pratiquant dans une perspective de progrès par des mobilisations particulières, voire de possibles innovations.

5. 4. Des propositions pour la diffusion

Dans cette optique, une telle démarche vise plus loin que la diffusion d'un article dans une revue scientifique. Sa véritable vocation est d'aller à nouveau «irriguer »le terrain. Dans cette dynamique, des artefacts (cognitifs, matériels, corporels ou réglementaires) sont élaborés et sont mobilisés. La concrétisation de la recherche technologique s'exprime alors au travers de la construction d'outils (schémas, matériels spécifiques, logiciels informatiques, etc.) ou de dispositifs (organisation d'une simulation, formalisation d'une situation...) qui deviendront peut-être des instruments pour le pratiquant (processus 


\section{eJRIEPS 23 avril 2011}

d'instrumentalisation). L'intervenant éducatif doit donc être en mesure de mobiliser voire de reconstruire de tels procédés. C'est à ce stade que nous envisageons l'articulation entre la recherche technologique sur les APSA et l'enseignement en technologie des APSA. En effet, le formateur de formateurs doit être à même de proposer des contextes de formation chargés de ces incidents afin de provoquer la construction de savoir qui vont au-delà de la reproduction de situations standardisées. Savoyant suggère que « le recours à des exemples de situations pratiques réelles (études de cas, situations reconstituées ou simulées) vise moins à développer une maîtrise pratique de ces situations spécifiques qu'à en repérer les savoirs qui en fondent l'organisation et en justifient la logique » (Savoyant, 2008, p. 99). Pour cette raison, les résultats des recherches technologiques sont utiles pour l'intervention en formation initiale et continue, voire dans les milieux professionnels. «II s'agit de développer des compétences préprofessionnelles, dans un environnement de recherche - intervention, qui valorisent sans les sacraliser les connaissances scientifiques, les savoirs technologiques et les intuitions personnelles conçues comme dynamiques et interactives » (Durey, 1995). II nous semblerait opportun que la formation en STAPS s'adosse à ces positions.

\section{Bibliographie}

Bouthier, D. (2005). Sciences et Techniques des APSA : quels rapports, vers quels métiers de l'intervention. Centre EPS et Société. Forum international de l'Education physique et du Sport. 4 -5-6 novembre 2005. Cité Universitaire Internationale de Paris. SNEP.

Bouthier, D. (2008 a). Technologie des APS : évolution des recherches et de leur place dans le cursus STAPS. eJRIEPS, 15, 44-59.

Bouthier, D. (2008 b). Hommage à René Deleplace In N. Wallian, M-P. Poggi \& M. Mussard (éds). Co-construire des savoirs : les métiers de l'intervention dans les APSA. Besançon : Presses Universitaires de Franche-Comté.

Bouthier, D., \& Reitchess, S. (Eds.). (1984). Contenus et évaluation en sports collectifs, pour un enseignement d'aujourd'hui. Paris, CRDP.

Clot, Y. (2003). La catachrèse entre réel et réalisé. Contribution d'un psychologue du travail. In Y.Clot \& R. Gori. La catachrèse : éloge du détournement. Nancy : PUN.

Cole, A. (1990). Cultural psychology, a once and future Discipline ?, Nebraska, Symposium.

Combarnous, M. (1094). Les techniques et la technicité. Messidor/Éditions sociales. 


\section{eJRIEPS 23 avril 2011}

Deleplace, R. (1966). Le Rugby. Analyse technique et pédagogique. Paris : Armand Colin. Deleplace, R. (1979). Rugby de mouvement rugby total. Paris: Ed. EPS.

Deleplace, R. (1983). La recherche sur la spécialisation sportive, l'entraînement, la performance. In Actes du colloque «la recherche en STAPS ». Nice. 19-20 septembre 1983, 93-151.

Durey, A. (1995). L’EPS et son rapport à la technique. Spirales, 8, 93-95.

Durey, A. \& Bouthier D. (1994). Technologie des activités physiques et sportives. Impulsions, 1, 117-126.

Eloi, S. (2009). Style d'un passeur de haut niveau en volley-ball. eJRIEPS, 17, 76-105.

Éloi, S., \& Uhlrich, G. (2001). Contribution à la caractérisation des sports collectifs : les exemples du volley-ball et du rugby. STAPS, 56, 109-125.

Galperine, P.I. (1980) (1966). Essai sur la formation par étapes des actions et des concepts In N. F. Talyzina (Ed.) De l'enseignement programmé à la programmation des connaissances. Lyon : PUL.

Garrassino, R. (1980). La technique maudite. Revue EPS, 164, 49-53.

Haudricourt, G. A. (1987). "L'origine des techniques». In La technologie, science humaine, recherche d'histoire et d'ethnologie des techniques. Paris. Maison des sciences de l'homme.

Jeu, B. (1983). Le sport, l'émotion, l'espace. Paris, Vigot.

Léontiev, A. (1974). The problem of activity in psychologie; Soviet Psychology, Winter 75, 13, 4-33.

Martinand, J.L. (1986). Connaître et transformer la matière. Bern : Peter Lang.

Martinand, J.-L. (1987). Quelques remarques sur la didactique des disciplines. Les sciences de l'éducation, 2, 23-35.

Martinand, J.L. (1994). La didactique des sciences et de la technologie et la formation des enseignants. ASTER, 19, 61-75.

Mouchet, A., Amans-Passaga, C. \& Gréhaigne, J.F. (2010). L'approche technologique, in M. Musard, M. Loquet \& G. Carlier (Eds.), Sciences de l'intervention en EPS et en sport : résultats de recherches et fondements théoriques, Paris, EPS, 201-222.

Norman, D. A. (1993). Les artefacts cognitifs. Raisons Pratiques, 4, 15-34.

Pastré, P. (1999). La conceptualisation dans l'action: bilan et nouvelles perspectives. Education Permanente, 139, 13-35.

Rabardel, P. (1993). Représentations dans des situations instrumentées. In A WeillFassina, P. Rabardel \& D. Dubois, Représentations pour l'action. (pp. 97-11). Toulouse : Ed. Octares. 


\section{eJRIEPS 23 avril 2011}

Rabardel, P. (1995). Les hommes et les technologies. Approche cognitive des instruments contemporains. Paris : Armand Colin.

Rabardel, P. \& Bourmaud, G. (2009). Instruments et systèmes d'instruments. In Rabardel, P. \& Pastré, P. (Eds). Modèles du sujet pour la conception - Dialectiques activités développement. (pp. 211-229). Toulouse :Octares.

Rabardel, P. \& Vérillon, P. (1985). Relations aux objets et développement cognitif. In Giordan, A. \& Martinand, J-L. (Eds). Actes des 70 journées internationales sur l'éducation scientifique. (pp. 189-196). Paris : LIRESPT, Université Paris VII.

Samurçay, R., \& Pastré, P. (1995). La conceptualisation des situations de travail dans la formation des compétences. Education Permanente, 125, 13-32.

Savoyant A. (2008). Quelques réflexions sur les savoirs implicites. Travail et apprentissages, 1, 92-100.

Savoyant A. (2010). Élément d'un cadre d'analyse de l'activité : quelques conceptions essentielles de la psychologie soviétique (1979). Travail et apprentissages, 5, 93107.

Sève, L. (1980). Une introduction à la philosophie marxiste. (2e ed.): Terrains/Editions sociales.

Trouche, L. (2005). Des artefacts aux instruments, une approche pour guider et intégrer les usages des outils de calcul dans l'enseignement des mathématiques. Actes de I'Université d'été de Saint-Flour. Le calcul sous toutes ses formes.

Uhlrich, G. \& Bouthier, D. (2008). Efficacité d'une situation de formation, par la simulation, à l'observation du mouvement général en rugby, eJRIEPS, 15,198-215.

Uhlrich, G., Éloi, S. \& Bouthier, D. (2008). La technologie dans le contexte des STAPS : de la conception d'outils à la conceptualisation... et réciproquement. eJRIEPS, 23.

Vergnaud, G. (1996). Au fond de l'action, la conceptualisation. In J.-M. Barbier(Eds.). Savoirs théoriques et savoirs d'action. (pp. 275-292). Paris : PUF.

Vérillon, P. (2005). Processus productifs et constructifs dans les activités physiques et sportives : la place de l'instrument. Impulsions, 4, 305-325.

Vigarello, G. (1991). Pour une technologie culturelle dans le champ des pratiques sportives. In J. Ardoino \& J.M. Brohm (Eds). Anthropologie du sport. Perspectives critiques. Actes du colloque international francophone. Paris-Sorbonne, 19-20 avril 1991. (pp. 146-151). Andsha - Matrice - Quel Corps ? 